\title{
Detection of a simulated gas leak in a wind tunnel
}

\author{
J Hodgkinson*1, Q Shan ${ }^{2}$ and R D Pride ${ }^{3}$ \\ Advantica Ltd, Ashby Rd, Loughborough, LE11 3GR, UK
}

\begin{abstract}
This paper brings together considerations of gas leak behaviour and leak detector design and use, with a view to improving the detection of low-pressure natural gas leaks. An atmospheric boundary layer wind tunnel has been used to study ground-based releases of methane at full scale over distances of up to $3 \mathrm{~m}$, under controlled conditions. These scales are relevant to the detection of natural gas leaks from mains and services using hand-portable gas detectors. The mean spatial distribution of the leaking gas plume was determined and used to test and fit a Gaussian dispersion model. This was used for subsequent analysis with respect to the ability of gas leak detectors to confirm and locate a leak. For ground-based leaks, gas concentrations drop rapidly with height such that instruments should ideally sample the air from within $10 \mathrm{~cm}$ of ground level. The rapid dilution of gas with distance from the source means that instruments with lower limits of detection, ideally of a few parts per million, have much improved ability to detect a leak from greater distances downwind. Finally, observations showed the variable temporal nature of the gas and the potential for confusion when sampling gas at a single point in time and space.
\end{abstract}

Keywords:

Gas leak, gas detection, methane, gas dispersion, wind tunnel

1 Now at Optical Sensors Group, School of Engineering, Cranfield University, Cranfield, Bedfordshire, MK43 0AL, UK. Address for correspondence.

2 Now at ABB Ltd, Gloucestershire, UK

3 Now at Institute for the Protection \& Security of the Citizen (IPSC), Joint Research Centre, European Commission, Ispra, Italy. 


\section{INTRODUCTION}

Accidental releases of natural gas from buried distribution pipes can have negative impacts on the environment and public safety. It has been estimated that approximately $90 \%$ of natural gas leakage (by volume) emanates from the low pressure distribution mains ${ }^{[1]}$. Gas distribution utilities worldwide therefore maintain emergency services to respond to public reported gas escapes (PREs), taking action to make safe any gas leaks ${ }^{[2]}$. The operator's tasks fall into the following simplified categories:

(i) Zero trace, or establishing the absence of gas, which can be the outcome for a significant fraction of PREs *.

(ii) Location of the ground - level leak source.

(iii) Quantification, especially on the \% LEL (lower explosive limit) concentration scale (outside the scope of this study).

The dispersion of accidental gas releases has been the subject of a considerable amount of investigation, giving rise to dispersion models based on several large-scale field studies in real conditions ${ }^{[4]}$. For passive gas releases (in which the gas has no significant momentum at the release point) the Pasquill - Gifford Gaussian model is often used (See Section 2 for details). Variants on this model include the work by Turner to quantify gas dispersion parameters over distances of $100 \mathrm{~m}-$ $100 \mathrm{~km}^{[5]}$.The motivation was to understand large-scale pollution rather than gas leak behaviour over the shorter length scales relevant to our study, and we were interested to understand whether this model could be usefully applied to the leak detection application described above. Earlier dispersion models have been found to have remarkable applicability to shorter length scales ${ }^{[4]}$.

There have been many studies to link the fluid dynamics of the process of gas detection with examples of chemical sensing in the animal kingdom. This field has been comprehensively reviewed by Weissburg ${ }^{[6]}$ and more recently by Settles ${ }^{[7]}$. However, there has been little work to explicitly link this understanding with the design of sensors for natural gas leaks from buried pipes.

A number of groups have developed autonomous gas detection robots to detect indoor and outdoor gas leaks. Lilienthal and Duckett have used a simple gas sensor array mounted on a robot, with dispersion modelling to estimate likely release locations ${ }^{[8]}$. Ishida et al have developed source location algorithms

\footnotetext{
* Of 1.35 M reported UK gas escapes in 2001, over 259,000 were outside reports, of which around $25 \%$ resulted in "no gas found" ${ }^{[3]}$.
} 
for a gas sensing robot that is able to move in response to the readings from its sensors ${ }^{[9]}$. In both cases the Gaussian dispersion model was used in a simplified form; a full understanding of characteristic levels of turbulence was not considered necessary. We have taken this a stage further by adopting the use of a more detailed dispersion profile based on experimental measurements. Instead of robotic detection algorithms, we have considered instrument design parameters and procedures for their use by human operators. The "zero trace" requirement is a particular feature of our application that has attracted little attention elsewhere.

We report a study of gas leak behaviour over short downwind distance of up to $3 \mathrm{~m}$, using a full-scale simulation of an outdoor low-pressure leak in a wind tunnel. These scales are relevant to leak detection activities for gas utilities' emergency response. The tunnel provided consistent conditions of wind speed, direction and turbulence over the course of the study.

Gas leaks can behave in a highly variable manner, both spatially and temporally, even under controlled conditions such as ours. We have considered the effects of sampling the gas concentration at different heights, in terms of the ability to zero trace and to locate a leak. We made time-averaged concentration measurements over a selected grid of downwind locations and then fitted these results to a Gaussian gas dispersion model that was used for subsequent analyses. Although we focused on the natural gas application described above, the work also has relevance to the detection and location of other gas releases.

\section{THEORY AND METHODOLOGY}

Figure 1 shows a schematic diagram of a gas release plume blown downwind of its source. We wanted to obtain a simple model to describe the time-averaged distribution of the plume, for subsequent analysis.

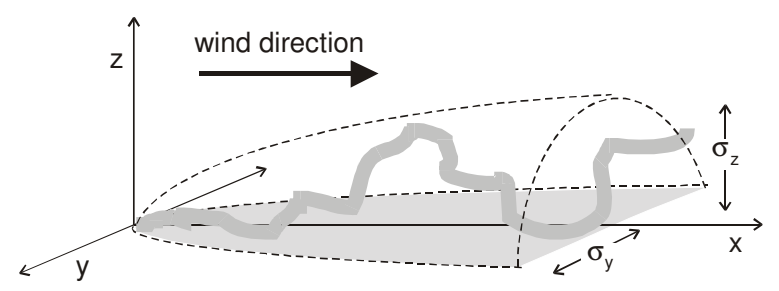

Figure 1. Schematic of a gas leak plume meandering within a defined envelope. 
In the Pasquill-Gifford model for passive gas releases, the concentration of gas downwind of a ground level, point leak source at the origin $(x, y, z)=(0,0,0)$ is predicted by the following Gaussian relationship ${ }^{[10]}$.

$$
C(x, y, z)=\frac{2 Q}{\pi \sigma_{y} \sigma_{z} u} \exp \left[-\frac{1}{2}\left(\frac{y^{2}}{\sigma_{y}^{2}}+\frac{z^{2}}{\sigma_{z}^{2}}\right)\right]
$$

$C$ is the concentration in $\mathrm{kg} \cdot \mathrm{m}^{-3}, Q$ is the flow rate in $\mathrm{kg} \cdot \mathrm{s}^{-1}, \sigma_{y}$ and $\sigma_{z}$ are dispersion coefficients in $\mathrm{m}$, $x, y$ and $z$ are distances in $\mathrm{m}$ and $u$ is the wind speed in $\mathrm{m} \cdot \mathrm{s}^{-1}$. For an extended source of width $2 a$ along the $y$-axis, equation (1) is integrated over the source:

$$
C(x, y, z)=\frac{Q}{\sqrt{2 \pi} \sigma_{z} u a} \exp \left(-\frac{1}{2} \frac{z^{2}}{\sigma_{z}^{2}}\right)\left\{\operatorname{erf}\left(\frac{1}{\sqrt{2}} \frac{(a-y)}{\sigma_{y}}\right)+\operatorname{erf}\left(\frac{1}{\sqrt{2}} \frac{(a+y)}{\sigma_{y}}\right)\right\}
$$

Ideally we would also integrate equation (2) over the source along the $x$-axis. However, the dependence of the dispersion coefficients, $\sigma_{y}$ and $\sigma_{z}$, on $x$ make this analytically difficult. Instead we have used equation (2) as it stands, making the approximation from a line source to our real rectangular source. At wind speeds of $\geq 1 \mathrm{~m} \mathrm{~s}^{-1}$ along the $\mathrm{x}$-axis, downwind dispersion over the short $(10 \mathrm{~cm})$ length of the source would be negligible.

Variants on this basic Gaussian model have been proposed for different conditions ${ }^{[10]}$, expressing $\sigma_{y}$ and $\sigma_{z}$ as different functions of $x$. One widely used model for the dispersion coefficients takes a simple power law, with alternative models converging to this relationship at shorter distances (down to $100 \mathrm{~m})^{[10]}$. We have therefore investigated the following widely used expression;

$$
\sigma=p x^{q}
$$

where $p$ and $q$ are determined experimentally for different stability conditions. Values given by Turner ${ }^{[11]}$ are given in Table 1, based on wind speed values measured at a height of $10 \mathrm{~m}$.

Table 1. Values of $\sigma_{y}$ and $\sigma_{z}$ given by Turner ${ }^{[11]}$.

\begin{tabular}{|c|c|c|c|}
\hline $\begin{array}{c}\text { Atmospheric stability } \\
\underline{\text { class }}\end{array}$ & $\sigma_{y}$ & $\sigma_{z}$ & $\begin{array}{c}\text { Example } \\
\text { occurrence }\end{array}$ \\
\hline Unstable (A) & $0.493 x^{0.88}$ & $0.087 x^{1.10}$ & $\begin{array}{c}\text { sunny days, low wind } \\
\text { speeds, warm ground }\end{array}$ \\
\hline Neutral (D) & $0.128 x^{0.90}$ & $0.093 x^{0.85}$ & no heat flux \\
\hline Stable (F) & $0.067 x^{0.90}$ & $0.057 x^{0.80}$ & $\begin{array}{c}\text { night time, low wind } \\
\text { speeds and cool ground }\end{array}$ \\
\hline
\end{tabular}




\subsection{Experimental set-up and methodology}

Our experimental configuration consisted of a simulated gas leak at full scale in a non-recirculating atmospheric boundary layer wind tunnel, capable of containing releases of explosive gases. The velocity profile is expected to show the following relationship for neutral conditions ${ }^{[12]}$;

$$
\frac{u(z)}{U^{*}}=\frac{1}{\kappa} \ln \left(\frac{z}{z_{0}}\right)
$$

Where $u$ is the wind speed, $\kappa$ is the von Karman constant (assumed equal to 0.4), $z$ is the height above ground level and $z_{0}$ is the surface roughness length (equal to approx $0.1 \times$ the height of roughness elements). $U^{*}$ is the friction velocity, a scaling factor that as a rule of thumb would be 0.05 to 0.1 times the wind speed at a height of $10 \mathrm{~m}$, the standard height used for measurements at airports. The relationship between a wind speed measured at height $z_{1}$ and one measured at $z_{2}$ is then given by,

$$
\frac{u_{1}}{u_{2}}=\ln \left(\frac{z_{1}}{z_{0}}\right) / \ln \left(\frac{z_{2}}{z_{0}}\right)
$$

A combination of surface roughness elements and boundary layer jets was used to establish a shear profile with a $z_{0}$ of approximately $1 \mathrm{~mm}$, representative of flat conditions, as shown in Figure 2. The profile was proportionally similar for wind speeds across the range used in our study $\left(1 \mathrm{~m} \mathrm{~s}^{-1}\right.$ $\left.2.5 \mathrm{~m} \mathrm{~s}^{-1}\right)$. A gas leak was constructed from a diffusing element $(140 \mathrm{~mm} \times 100 \mathrm{~mm})$ at ground level, releasing methane into the air at known leak rates. Two values of $\mathrm{z}_{0}$ are plotted for comparison; a value of $z_{0}=1 \mathrm{~mm}(+2 \mathrm{~mm} /-0.7 \mathrm{~mm}$, ie between $3 \mathrm{~mm}$ and $0.3 \mathrm{~mm})$ showed the best fit to the data. However, for heights in the region $0-200 \mathrm{~mm}$ (of greatest interest in this study) measurements were difficult to make and we have limited data.

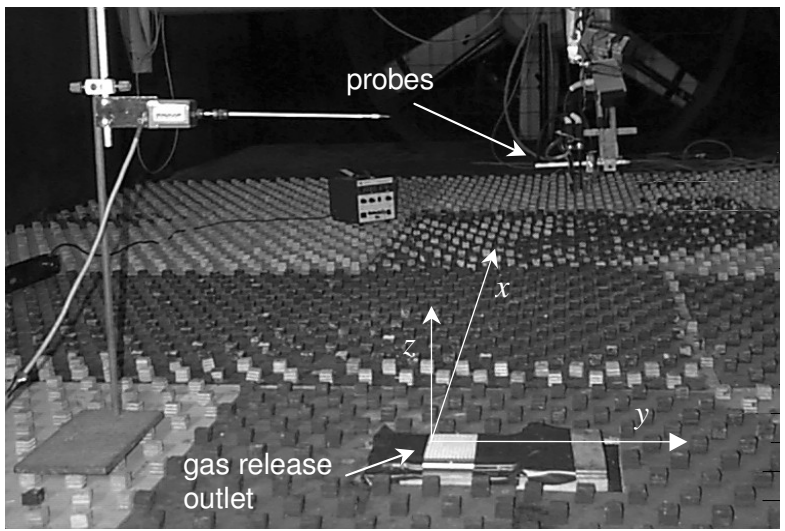

(a)

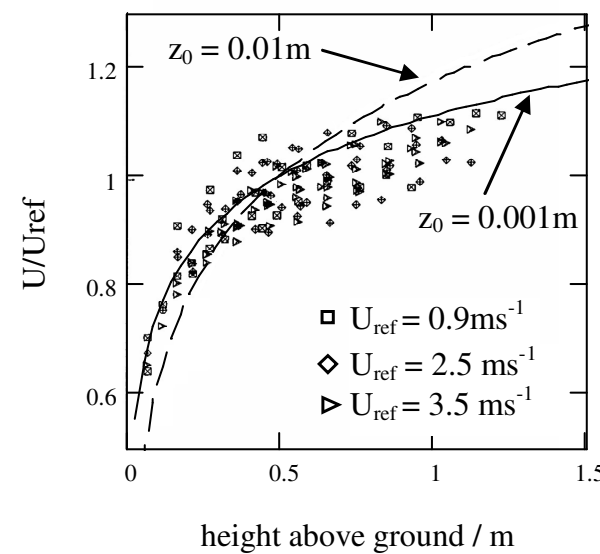

(b)

Figure 2. Wind tunnel layout (a) showing the gas release point and instrument probes. Boundary layer jets and roughness elements on the floor provided a controlled velocity profile (b). 
Gas concentrations were measured downwind of the source with different gas detectors, their sample probes mounted on a three axis translation arm, to allow measurements over a space $2 \mathrm{~m}$ wide $\times 1 \mathrm{~m}$ high $\times 3 \mathrm{~m}$ downwind of the leak. Three gas detectors were used: a Telegan Gastec flame ionisation detector (FID), a GMI Gasurveyor employing a pellistor (catalytic sensor) on the ppm and \%LEL range, and a Cambustion HFR400 fast-response FID. During the leak experiments the wind speed was controlled and monitored at a height of $1 \mathrm{~m}$ above ground level, which was considered high enough to avoid perturbing the flow in the region of the sensors.

\section{EXPERIMENTAL RESULTS}

\subsection{Observations of transient behaviour}

The leaking gas was first assessed qualitatively using smoke visualisation. Smoke was introduced to the gas leak point and allowed to track in the wind tunnel, where its behaviour was recorded using a bright lamp and video camera. A good correlation between the density of a smoke plume and the concentration of methane released in similar conditions has been established for line-of-sight integrated measurements by Nappo ${ }^{[13]}$, and for point measurements by Yamanaka et al ${ }^{[14]}$.

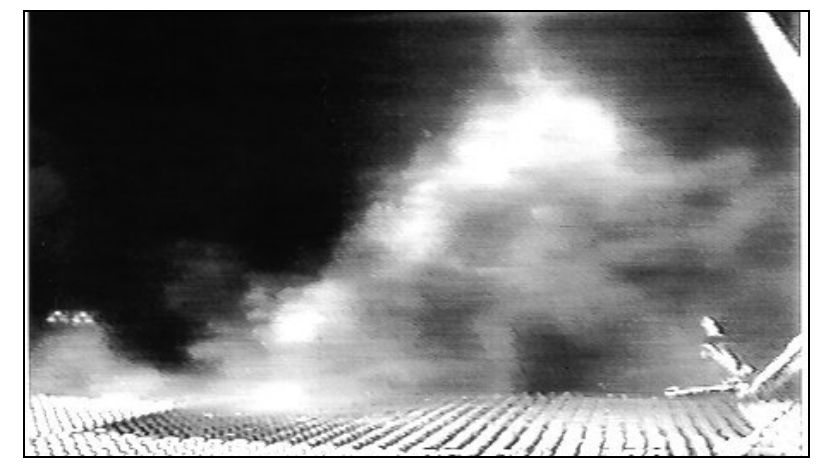

Figure 3. Smoke leak used as a natural gas analogue, showing a spatially variable smoke concentration.

Transient behaviour was quantitatively assessed using all three instruments at a fixed point. Time series readings from the different instruments were collected simultaneously, with the sample inlets of both detectors located as close as possible to one another (within approximately $5 \mathrm{~cm}$ ). It was confirmed that measurements made using one probe were not affected by the proximity of the others. An example of the results is shown in Figure 4. 
The Cambustion instrument had the fastest response time of $0.25 \mathrm{~s}$, and demonstrated that in some locations the gas was present in sharp spikes of high concentration. It is not known whether at $0.25 \mathrm{~s}$ these spikes were fully resolved, or whether with shorter response times, narrower peaks would be detected; other studies have found evidence for fractal structures in dispersing gas plumes ${ }^{[15]}$.

The Telegan FID showed an initially delayed response, consistent with a long sampling tube, and a subsequent response time of a few seconds. The GMI Gasurveyor showed a further delay plus long response time totalling $>5 \mathrm{~s}$. The distance between the sample points was too small, at wind speeds of $\geq 1 \mathrm{~m} \mathrm{~s}^{-1}$, to account for these delays.

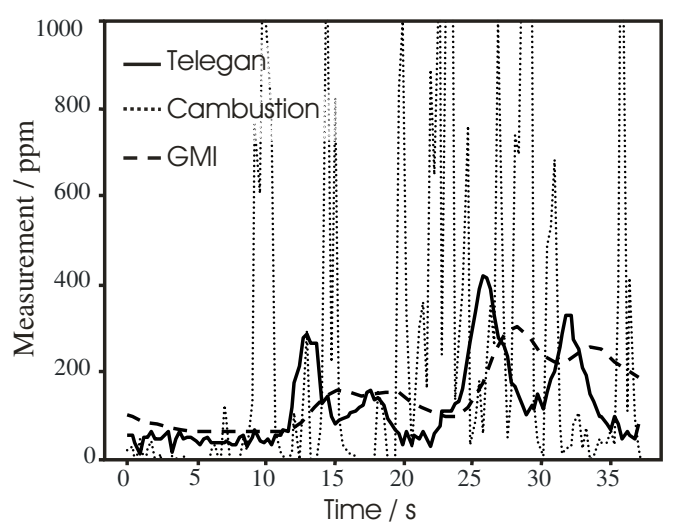

Figure 4. Comparison of time series measurements from three gas detectors. Example taken at $(x, y, z)=(1 \mathrm{~m}, 200 \mathrm{~mm}, 20 \mathrm{~mm})$ with a wind speed of $1.8 \mathrm{~m} \mathrm{~s}^{-1}$.

An instrument needs a fast response time in order to positively respond to small, thin wisps of gas as they pass by. Our results show that response time differences in the range $0.25 \mathrm{~s}-5 \mathrm{~s}$ give noticeably different results, and we conclude that short (of the order of $1 \mathrm{~s}$ or less) response times would be preferred in this application. Instruments with slower response times would need an improved limit of detection to establish a reliable zero trace. Thus there is a trade-off between response time and limit of detection because of the variability of the measurand. Furthermore, sampling delays have the potential to confuse the operator, since the sampling probe will have moved by up to $2 \mathrm{~m}$ within a delay period of approximately $2 \mathrm{~s}$. Another study has confirmed this problem for robotic gas detection $\operatorname{algorithms}^{[16]}$.

The transient behaviour of the gas was found to vary from one location to another, as illustrated in Figure 5. A useful clue to the leak's location might be found within this information, either in its raw form or possibly correlated with measurements of wind speed and direction ${ }^{[17]}$. 


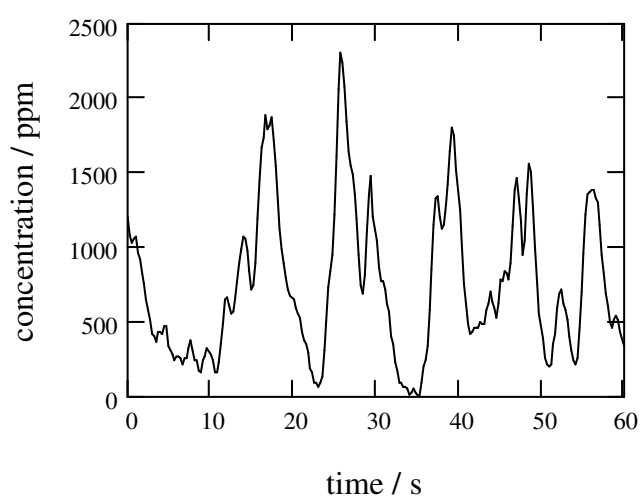

(a) $(x, y, z)=(250 \mathrm{~mm}, 0 \mathrm{~mm}, 50 \mathrm{~mm})$

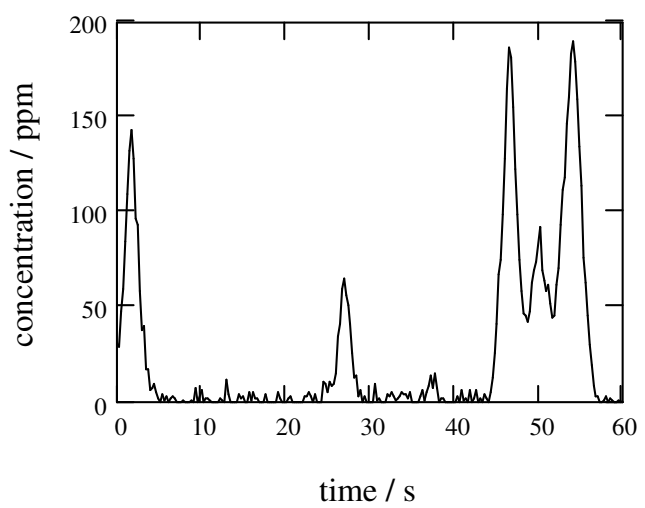

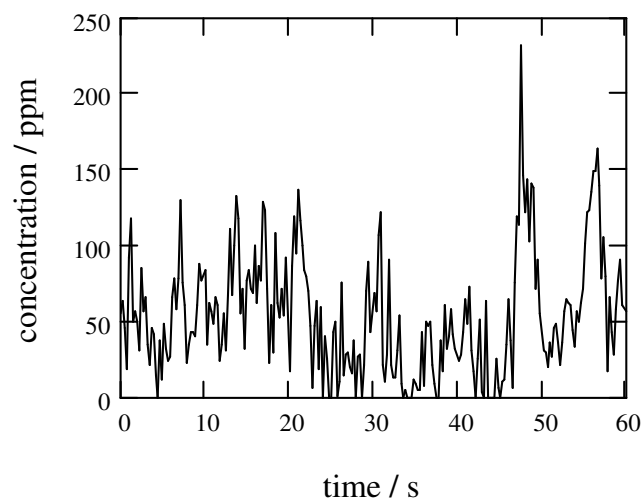

(b) $(\mathrm{x}, \mathrm{y}, \mathrm{z})=(3 \mathrm{~m}, 0 \mathrm{~mm}, 200 \mathrm{~mm})$

(c) $(\mathrm{x}, \mathrm{y}, \mathrm{z})=(3 \mathrm{~m}, 500 \mathrm{~mm}, 200 \mathrm{~mm})$

Figure 5. Transient behaviour of the gas in different locations, as measured by a Telegan FID in a wind speed of $1 \mathrm{~m} \mathrm{~s}^{-1}$. Examples shown (a) close to the source, $(b)$ in an intermediate zone, (c) at the extremes of the plume.

The results in Figure 5 are consistent with the gas forming a series of wisps, possibly caused by the meandering of one or more plumes. Close to the leak, the presence of gas is unambiguous. However, at the extremes it is possible that a gas detector might measure no gas concentration above the background. This statistical likelihood is unquantified in our study, but visible in the separation between peaks of gas concentration, which could last over $10 \mathrm{~s}$ (see Figure $5 \mathrm{c}$ ). The consequence is that zero tracing using a point sampling gas detector would need to take longer, and we cannot rely on our time-averaged concentrations as an absolute guide to confidence in instantaneous zero readings. Further, statistical analysis of our data is recommended to quantify this observation. 


\subsection{Distribution of downwind gas concentration}

Gas concentrations were measured and averaged over two minutes at a range of locations. For quantitative work, the Telegan Gastec was used as it provided a low (5 ppm) limit of detection and is in widespread use for investigating outdoor gas leaks. Checks were made that the averaged gas concentration varied proportionally with leak rate and with initial concentration, as predicted by equation (2), enabling us to normalise our results to a standard leak rate of $100 \%$ volume at 1 litre $\min ^{-1}$.

It was also confirmed that the gas dispersion profile was symmetric about the centre plane $y=0$, allowing representative measurements to be made over only half the experimental space. Concentrations predicted by equation (2) were compared to the measured concentrations for each of the Pasquill stability classes given in Table 1. Agreement between the two was poor so we developed a simple empirical model following the same form, fitting new values for dispersion coefficients using a least squares approximation.

We considered two options with our model, as follows.

(i) For simplicity, we first chose to use values of $u$ in equation (2) corresponding to the reference wind speeds monitored in the wind tunnel at a height of $1 \mathrm{~m}$, denoted $u_{l}$. This had the advantage of not making our model unnecessarily dependent on an estimate of the roughness length $z_{0}$, for which we had limited data.

(ii) In the second option we chose to use values of $u$ in equation (2) corresponding to the value that would be measured at a height of $10 \mathrm{~m}$ in equivalent conditions, following equation (5). This has been denoted $u_{10}$ and calculated using our previous estimate of $z_{0}=1 \mathrm{~mm}$. For $u_{1}=1 \mathrm{~m} \mathrm{~s}^{-1}, 1.8 \mathrm{~m} \mathrm{~s}^{-1}$ and $2.5 \mathrm{~m} \mathrm{~s}^{-1}$, the corresponding values for $u_{10}$ are $1.3 \mathrm{~m} \mathrm{~s}^{-1}, 2.4 \mathrm{~m} \mathrm{~s}^{-1}$ and $3.3 \mathrm{~m} \mathrm{~s}^{-1}$, respectively.

A different fit was established for each wind speed, giving the $\sigma$ values shown in Table 1 . The $\sigma_{y}$ and $\sigma_{z}$ values in Table 2 (ii) are directly comparable with those of Turner in Table 1. Table 3 shows the rms error for our data points with a Gaussian distribution based on each of the dispersion coefficients.

Agreement with both cases of the new fitted model was better than with the models based on Turner's original parameters. The large error levels are attributed to variability of the measurand. The measured concentration at each point represents a numerical mean of two minutes of time series data, with an associated standard deviation of between $50 \%$ of mean value (more typical of data taken along the centre line) to between $100 \%$ and $120 \%$ of the mean value (more typical of data taken away from the 
centre line). The standard error in the mean at each point is dependent on the number of independent samples taken over two minutes. Approximately 500 data points were recorded in each case, but as consecutive data points were correlated, we cannot estimate the error in a single time-averaged concentration value. However, given the large standard deviations in the underlying data, it is perhaps not surprising that the rms difference between the modelled concentration and that recorded at each data point might be no better than $50-60 \%$ of the overall mean concentration.

Table 2. Values of $\sigma_{y}$ and $\sigma_{z}$ as used in this study.

Basis of model:

(i) Reference $u$ values at $1 \mathrm{~m}$ height

\begin{tabular}{|c|c|c|}
\hline$\underline{u_{l}} \frac{/ \mathrm{m} \mathrm{s}^{-1}}{\sigma_{y}}$ & $\sigma_{z}$ \\
\hline 1 & $0.14 x^{0.10}$ & $0.21 x^{0.97}$ \\
\hline 1.8 & $0.15 x^{0.39}$ & $0.16 x^{0.93}$ \\
\hline 2.5 & $0.14 x^{0.54}$ & $0.12 x^{0.94}$ \\
\hline
\end{tabular}

Basis of model:

(ii) Equivalent $u$ values at $10 \mathrm{~m}$ height

\begin{tabular}{|c|c|c|c|}
\hline$\underline{u_{1}} / \mathrm{m} \mathrm{s}^{-1}$ & $\underline{u_{10}} / \mathrm{m} \mathrm{s}^{-1}$ & $\sigma_{y}$ & $\sigma_{z}$ \\
\hline 1 & 1.3 & $0.13 x^{0.20}$ & $0.15 x^{0.85}$ \\
\hline 1.8 & 2.4 & $0.13 x^{0.55}$ & $0.13 x^{0.81}$ \\
\hline 2.5 & 3.3 & $0.11 x^{0.68}$ & $0.10 x^{0.87}$ \\
\hline
\end{tabular}

Table 3. RMS errors for the extended source model, calculated using the fitted and original dispersion coefficients given in Table 1.

\begin{tabular}{|c|c|c|c|c|c|c|}
\hline \multirow{3}{*}{$\begin{array}{c}u_{1} \\
/ \mathrm{m} \mathrm{s}^{-1}\end{array}$} & \multicolumn{5}{|c|}{ rms error / ppm } & \multirow{3}{*}{$\begin{array}{l}\text { Mean measured } \\
\text { concentration (for } \\
\text { comparison) } \\
\text { / ppm }\end{array}$} \\
\hline & \multicolumn{2}{|c|}{ fitted model (Table 2) } & \multicolumn{3}{|c|}{ Turner's model (Table 1) } & \\
\hline & (i) using $u_{l}$ & (ii) using $u_{10}$ & $\begin{array}{c}\text { stable } \\
\text { (A) }\end{array}$ & $\begin{array}{c}\text { neutral } \\
\text { (D) }\end{array}$ & $\begin{array}{l}\text { unstable } \\
\text { (F) }\end{array}$ & \\
\hline 1 & 73 & 49 & 197 & 242 & 389 & 91 \\
\hline 1.8 & 42 & 37 & 171 & 117 & 214 & 68 \\
\hline 2.5 & 29 & 34 & 136 & 51 & 38 & 55 \\
\hline
\end{tabular}

Lees has discussed the validity of various dispersion models ${ }^{[4]}$ and points out that Turner's dispersion coefficients were considered estimates, expected to be within a factor of 2 of the true values. Our empirically determined values fell within this expectation, apart from the value of $\sigma_{y}$ for the $1 \mathrm{~m} \mathrm{~s}^{-1}$ experiment, for which we have a reduced $x$-dependence of compared to Turner's figures. 
Turner's dispersion coefficients are understood to have been established for outdoor experiments over distances of greater than $100 \mathrm{~m}$, and we are therefore uncertain as to the validity of this model much closer to the leak source. Furthermore, in our experiments the gross wind direction was constrained, however in an outdoor test the wind direction will have been able to change during the experiment, resulting in a wider apparent plume for time-averaged data. Previous studies have shown that plume variance $\sigma_{y}^{2}$ can be considered the sum of two variances, resulting from (i) concentration fluctuations around the instantaneous centre line, and (ii) a second variance resulting from meandering of the centre line ${ }^{[4]}$. We could therefore expect that unconstrained experiments would generally result in wider plumes and larger values of $\sigma_{y}$. Our results all showed reduced x-dependence of $\sigma_{y}$ and slightly different plume shapes at each wind speed.

It is noteworthy that the fitted dispersion model is different for our two cases, based on the arbitrary choices of $1 \mathrm{~m}$ and $10 \mathrm{~m}$ reference heights for wind speed measurements. Examination of equations (2) and (5) indicates that using different reference heights for wind speeds gives a simple scale change in the value of $u$, but to best fit the data, slightly different choices of $\sigma_{y}$ and $\sigma_{z}$ would be needed in each case, resulting in different shaped plumes. This perhaps indicates the shortcomings of using a relatively simple model in this work, the more so with a highly variable measurand where even our time-averaged data were subject to an unquantified (but believed to be proportionally high) level of uncertainty.

Despite these shortcomings, our approach has been to use the fitted dispersion models as representations of the particular circumstances of our wind tunnel experiments. The dispersion coefficients thus encapsulate our readings to form a simple empirical model, with which we can examine trends in the data. In gas leak detection applications, the circumstances surrounding each leak will anyway be different in each case, and this needs to be noted when interpreting the results, regardless of the degree of sophistication of the model.

\section{VISUALISATION AND INTERPRETATION}

The fitted model obtained above was used as a 3-D (3-dimensional) gas concentration distribution for the following analyses, using different representations of the data. We chose to represent the data using the second model, using $u_{10}$, as it gave a generally better fit to our data across all 3 wind speeds.

\subsection{Limits of detection}

We can establish regions within which leaks are detectable for different instrumental limits of detection (lod), chosen to be representative of instruments in use. Although as previously discussed a 
statistical analysis is needed to establish an absolute level of confidence in a zero trace, we can nevertheless observe trends between instruments with different lods. For illustrative purposes we can compare the cases of a 5 ppm lod shown in Figure 6 (a), and a 250 ppm lod shown in Figure 6 (b). The values of $5 \mathrm{ppm}$ and $250 \mathrm{ppm}$ were chosen to be representative of the Telegan FID $^{[18]}$ and a pellistorbased instrument ${ }^{[19]}$.
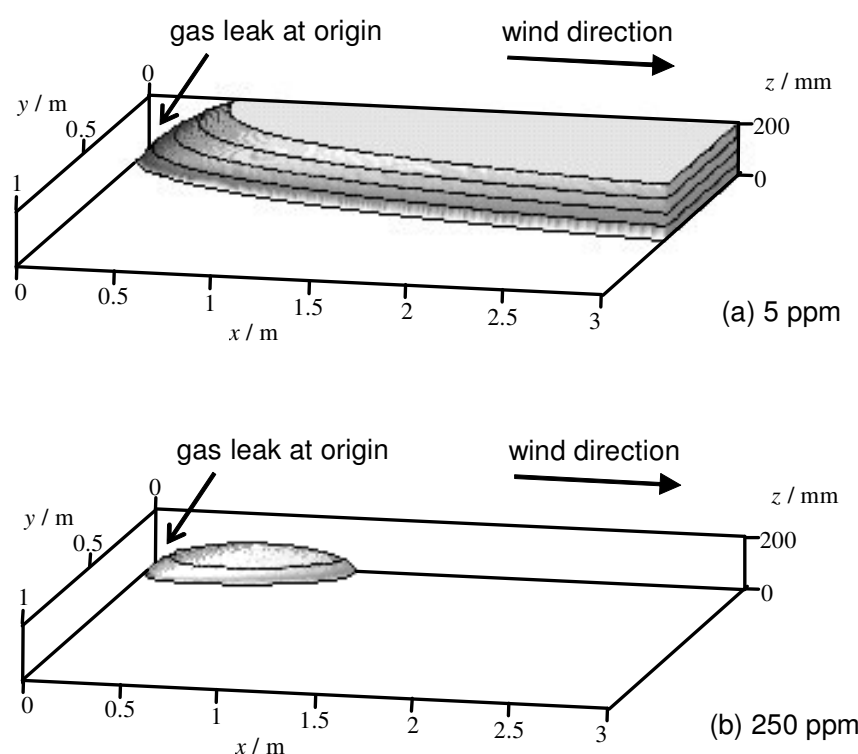

Figure 6. Modelled isopleths for a wind speed of $1.8 \mathrm{~m} \mathrm{~s}^{-1}$, at a concentration of (a) 5 ppm and (b) 250 ppm. Contours are shown at intervals of $z=50 \mathrm{~mm}$.

In each case the plot shows an isopleth at a concentration equal to the lod. Within the enclosed volume we can consider the gas leak to be reliably detectable, or else this is the volume within which a zero reading would give an accurate zero trace for a 1 litre $\mathrm{min}^{-1}$ leak at the origin. Clearly, instruments with lower lods can lead to more reliable and / or broader indications of zero trace. The ability to detect gas downwind of a leak at larger distances is also an indication that an area could be surveyed with greater efficiency. The plots cannot however be used to develop guidelines for instantaneous measurements because concentration measurements were averaged over periods of 2 minutes.

\subsection{Effects of sampling height}

Equation (1) predicts that gas concentration reduces with increasing height. Operators should be familiar with the need to sample gas close to ground level and a number of sampling probe accessories are marketed for this purpose. Information available to the operator can be represented as a 2-D slice through our data at constant height $\mathrm{z}$, as shown in Figure 7. 

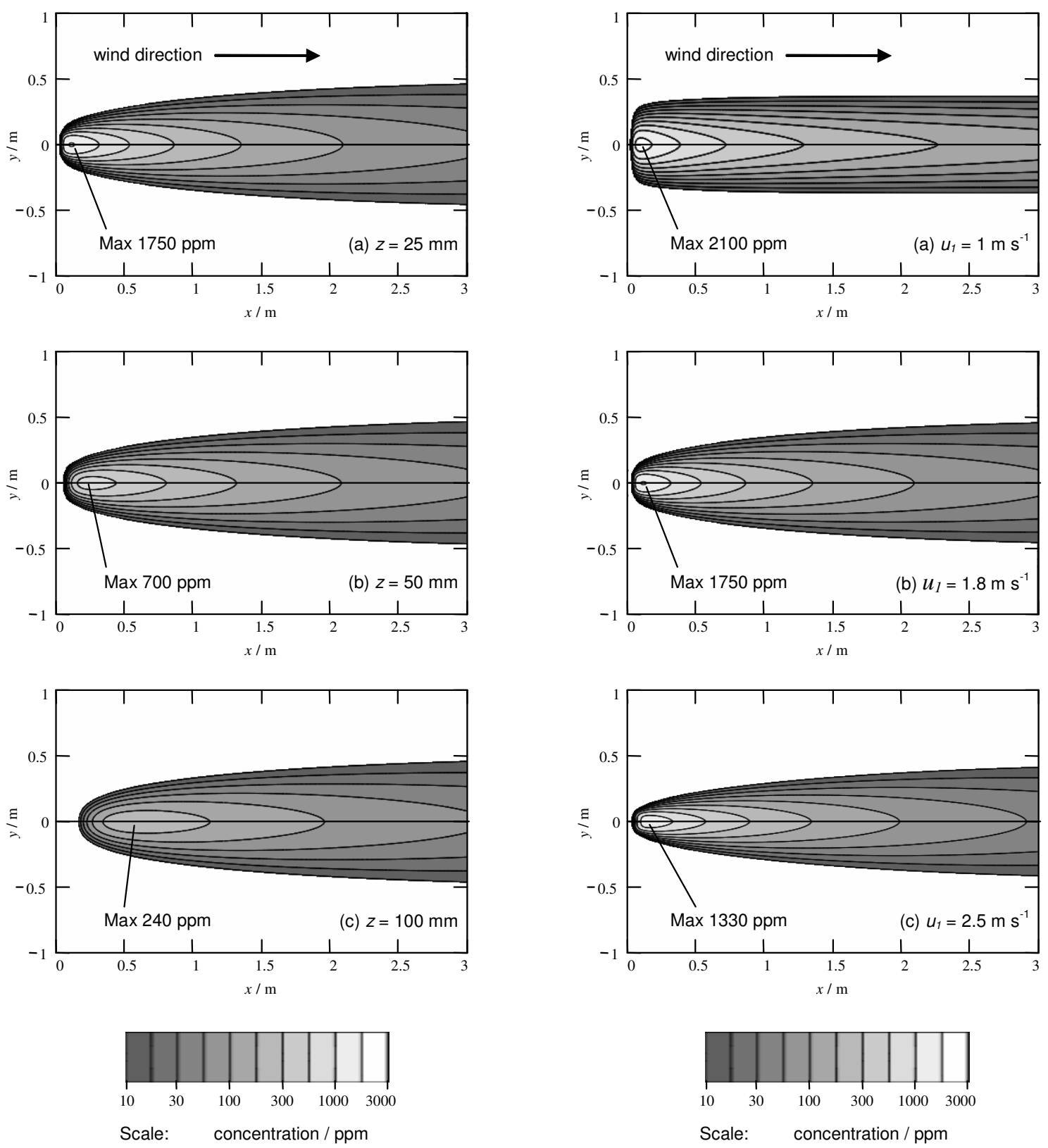

(i) Effect of sampling height at constant wind

$$
\text { speed }\left(u_{1}=1.8 \mathrm{~m} \mathrm{~s}^{-1}\right) \text {. }
$$

(ii) Effect of different wind speeds $u_{1}$ at constant sampling height $(z=25 \mathrm{~mm})$.

Figure 7. Modelled concentration contours downwind of a 1 litre min $^{-1}$ leak The contours have been truncated at 10 ppm for clarity.

The following observations can be made. Our investigations show that these general points are not dependent on the precise form of the model used in section 3.2.

(i) These experiments confirm that the gas concentration drops rapidly with increasing height, quantified by low values of $\sigma_{z}$ in Table 2 (between 100 and $150 \mathrm{~mm}$ at a downwind distance $x$ of $1 \mathrm{~m}$, for case (ii)). Figure 7 illustrates that for best performance in both detection and leak 
location activities, sampling heights should be below $100 \mathrm{~mm}$ and preferably as low as possible (around $25 \mathrm{~mm}$ ). This conclusion applies to the flat ground conditions of the study; the possible effect of increased turbulence caused by undulations is not quantified.

(ii) At greater sampling heights the source would be more difficult to locate; the width of the peak in gas concentrations is broader and that peak is further downwind from the ground-based source. Even at a height of only $100 \mathrm{~mm}$ above ground, the concentration peak can be $>0.5 \mathrm{~m}$ from the source.

(iii) Peak concentrations are low compared to the initial concentration of the leak (100\% volume) and the gas would be even more dilute in stronger winds; in the lightest wind studied $\left(u_{l}=1 \mathrm{~m} \mathrm{~s}^{-1}\right)$ the mean concentration was diluted by a factor of approximately 500 at the peak when measured at a sampling height of $25 \mathrm{~mm}$.

\section{DISCUSSION AND CONCLUSIONS}

This study has taken results from a gas leak in simulated wind conditions and interpreted them in relation to gas leak detection operations. We can draw out a number of conclusions that relate to the activities of leak detection and zero tracing.

(i) Over flat ground, zero tracing and leak location are best conducted with a sample probe as close to the ground as possible, at heights of under $100 \mathrm{~mm}$ and preferably as low as $25 \mathrm{~mm}$.

(ii) Measuring a zero trace at a particular location implies that the gas concentration is also zero in a thin strip upwind of that location, for a 2 minute time-averaged measurement. It implies little about the gas concentration downwind or more than perhaps $\pm 300 \mathrm{~mm}$ or $\pm 500 \mathrm{~mm}$ crosswind (depending on the distance to the source). An awareness of wind conditions in the region of the sample probe could therefore enhance operators' effectiveness.

(iii) The measurand is highly variable at a single sampling location, such that instruments with response times of the order of 1-2 s have an advantage when detecting low concentrations that appear in narrow bursts.

(iv) In stable conditions with a more or less constant wind direction and wind speeds within our experimental range, the following would be a good strategy for locating a single leak source. A similar algorithm has been reported by Ishida et al for their autonomous robotic gas detectors ${ }^{[9]}$

- Tracking across the wind, find the position of the highest gas concentration.

- Move upwind by up to $1 \mathrm{~m}$ from this position, and repeat. (In practice, operators habitually swing the probe arm in a zig-zag fashion, with the same effect.) 
- The source is likely to be upwind of the highest measured concentration (for example by $50-100 \mathrm{~mm}$ in our study).

Our results help to explain why zero tracing and leak location are difficult to complete using instruments that measure gas at a single point in time and space. The variability of the gas plume and the response time of an instrument (of up to a few seconds) can conspire to confuse operators about the presence of gas at a particular location and the whereabouts of the leak source.

\section{ACKNOWLEDGEMENTS}

We would like to thank National Grid Transco and BG Asia Pacific for their support. The latter funded three students who assisted with the work, namely Soo San Chim, Ivy Ai Ling Lee and Siti Rohaiyah Tumpang. We would also like to thank John Smith and Olric Morrison for their help with data processing.

\section{REFERENCES}

[1] Williams A (Editor) 1994 Methane emissions Report of Watt Committee on Energy 28

[2] Institution of Gas Engineers and Managers 1998 Dealing with reported gas escapes. IGEM SR/20 Edition 2 (London: IGEM)

[3] Transco website 2005 http://www.transco.co.uk/safety/index/index.asp

[4] Lees FP 1996 Loss Prevention in the Process Industries (Butterworth-Heinemann)

[5] Turner DB 1970 Workbook of atmospheric dispersion estimates Office of Air Programs (NC, USA: EPA)

[6] Weissburg MJ 2000 The fluid dynamical context of chemosensory behavior Biol. Bull. 198 188202

[7] Settles GS 2005 Sniffers: Fluid-dynamic sampling for olfactory trace detection in nature and homeland security - the 2004 Freeman Scholar lecture J. Fluids Engineering 127 189-218

[8] Lilienthal A and Duckett T 2004 Building gas concentration gridmaps with a mobile robot Robotics and Autonomous Systems 48 3-16

[9] Ishida H, Nakamoto T and Moriizumi T 1998 Remote sensing of gas / odor source location and concentration distribution using mobile system Sensor. Actuat. B-Chem 49 52-57 
[10] Lees FP 1996 Loss Prevention in the Process Industries (Butterworth-Heinemann)

[11] Turner DB 1970 Workbook of atmospheric dispersion estimates Office of Air Programs (NC, USA: EPA)

[12] Hanna SR and Britter RE 2002 Overview of meteorology and atmospheric dispersion, Chapter 2 in Wind flow and vapour cloud dispersion at industrial and urban sites (AIChE)

[13] Nappo CJ 1984 Turbulence and dispersion parameters derived from smoke-plume photoanalysis Atmos. Environ. 18 299-306

[14] Yamanaka T, Ishida H, Nakamoto T and Moriizumi T 1998 Analysis of gas sensor transient response by visualizing instantaneous gas concentration using smoke. Sensor. Actuat. A-Phys 69 $77-81$.

[15] Hadad A, Stiassnii M, Poreh M and Cermak JE 1993 Fractal aspects of integrated concentration fluctuations Bound.-Lay. Meteorol. 62 291-302.

[16] Ishida H, Nakayama G, Nakamoto T and Moriizumi T 2002 Controlling a gas / odor plumetracking robot based on transient responses of gas sensors Proc IEEE Sensors 2002, Orlando, Florida 1665-1670

[17] Shan Q and Pride R 2000 Portable apparatus and method for tracing a gas leak Patent application WO 0073761

[18] National Grid Transco 1984 Transco Technical Specification For Portable Instruments Of High Sensitivity To Detect Natural Gas. Publication INQ4 (Warwick, UK: NGT)

[19] National Grid Transco 1995 Transco Technical specification for Portable Gas Detectors. Publication INQ3 (Warwick, UK: NGT) 